\title{
Gradhiva
}

GRADHI

Revue d'anthropologie et d'histoire des arts

26 | 2017

En croire ses sens

\section{Entretien avec Jean-Claude Schmitt}

22 mars 2017

Jean-Claude Schmitt, Giordana Charuty et Emma Aubin-Boltanski

\section{CpenEdition}

Journals

Édition électronique

URL : http://journals.openedition.org/gradhiva/3464

DOI : 10.4000/gradhiva.3464

ISSN : 1760-849X

Éditeur

Musée du quai Branly Jacques Chirac

Édition imprimée

Date de publication : 6 décembre 2017

Pagination : 164-179

ISBN : 978-2-35744-096-8

ISSN : 0764-8928

Référence électronique

Jean-Claude Schmitt, Giordana Charuty et Emma Aubin-Boltanski, « Entretien avec Jean-Claude

Schmitt », Gradhiva [En ligne], 26 | 2017, mis en ligne le 06 décembre 2019, consulté le 14 septembre 2020. URL : http://journals.openedition.org/gradhiva/3464

(c) musée du quai Branly 


\section{Entretien avec Jean-Claude Schmitt 22 mars 2017}

par Giordana Charuty et Emma Aubin-Boltanski

Gradhiva - Le recueil d'articles que vous publiez en 2001, Le Corps, les rites, les rêves, le temps, a pour sous-titre Essais d'anthropologie médiévale 1. Dans la préface, vous récusez d'emblée la catégorie de "religion", au profit de celle de culture et de système symbolique. Comment ce choix s'est-il imposé?

Jean-Claude Schmitt (J.-C. S.) - Longtemps, I'histoire religieuse a été écrite par des clercs, y compris au CNRS jusqu'à une date récente. La religion était étudiée à travers le prisme de l'engagement confessionnel. D'où, en particulier, l'efflorescence des travaux sur la «religion populaire» qui accompagnèrent le mouvement conciliaire. L'Église se penchait sur le peuple, pour découvrir que celui-ci avait des croyances en porte à faux, voire dégradées (on parla aussi de «survivances» de «croyances païennes»), par rapport au magistère et à la théologie. Mon intérêt pour les sciences sociales voisines et notamment pour l'anthropologie m'invitait à poser autrement les questions, dans les termes - systèmes symboliques, culture - que vous venez de rappeler.

\section{G. - Quels étaient, alors, vos principaux interlocuteurs?}

J.-C.S. - J'ai eu une formation un peu complexe, partagée entre ce temple de la tradition érudite qu'était l'École des chartes et le séminaire de Jacques Le Goff, qui m'a ouvert à l'anthropologie historique. Entretemps, j'avais fait ma maîtrise à la Sorbonne avec Michel Mollat, qui était un homme délicieux, soucieux de donner une dimension sociale à l'histoire religieuse. J'ai sous sa direction transformé en thèse de doctorat ma thèse de l'École des chartes, sur les béguines et les béguards du Rhin supérieur ${ }^{2}$. Ce n'était pas de l'histoire religieuse, mais de l'histoire sociale: je me demandais comment avait évolué pendant deux siècles le discours polémique visant des groupes «marginaux " considérés d'abord comme des hérétiques, puis comme des parasites sociaux, quand les tensions sur le marché de la main-d'œuvre poussaient à les forcer à travailler de leurs mains. Mon sujet n'était pas du tout celui, classique dans ces années-là, de la spiritualité des béguinages. J'étais plutôt, peu après 1968, influencé par l'histoire et la sociologie de la marginalité sociale, telles qu'elles se pratiquaient à Jussieu notamment, où Bernard Vincent et d'autres conduisaient une enquête sur les marginaux et les exclus dans I'histoire ${ }^{3}$. Bronislaw Geremek avait ouvert la voie ${ }^{4}$. Mon deuxième livre, Le Saint lévrier ${ }^{5}$, traduit un changement radical de perspective dû à ma découverte de l'ethnologie. Celle-ci s'est faite un peu par hasard - pour autant que le hasard existe en ces domaines -, à travers l'enquête sur les exempla initiée par Jacques Le Goff et poursuivie aujourd'hui par Marie-Anne Polo de Beaulieu et Jacques Berlioz ${ }^{6}$. Ces récits brefs mis par écrit et utilisés par les prédicateurs ont été largement dédaignés
1. Jean-Claude Schmitt, Le Corps, les rites, les rêves, le temps: essais d'anthropologie médiévale. Paris, Gallimard, 2001.

2. Jean-Claude Schmitt, Mort d'une hérésie: l'Église et les clercs face aux béguines et aux béghards du Rhin supérieur, du xiv ${ }^{e}$ au $\times v^{\ominus}$ siècle. Paris/ La Haye/New York, Mouton/EHESS, 1978.

3. Bernard Vincent (dir.), Les Marginaux et les exclus dans l'histoire. Paris, 10/18-UGE, 1979.

4. Bronislaw Geremek, Les Marginaux parisiens aux XIV et $\times v^{e}$ siècles, trad. du polonais par Daniel Beauvois. Paris, Flammarion, 1976.

5. Jean-Claude Schmitt, Le Saint lévrier: Guinefort, guérisseur d'enfants depuis le $x{ }^{\prime \prime}{ }^{\circ}$ siècle. Paris, Flammarion, 2004 [1979].

6. Claude Brémond Jacques Le Goff et Jean-Claude Schmitt, L'Exemplum, Turnhout, Brepols, 1982 ; Jacques Berlioz et Marie-Anne Polo de Beaulieu (dir.), Les Exempla médiévaux nouvelles perspectives, actes du Colloque international de Saint-Cloud (1994). Paris, Honoré Champion, 1998. 
7. Vladimir Propp, Morphologie du conte, trad. du russe par Marguerite Derrida Claude Kahn,

Tzvetan Todorov. Paris, Seuil, 1965 [1928].

Voir la critique de Claude Lévi-Strauss: "La structure et la forme. Réflexions sur un ouvrage de Vladimir Propp ", in Anthropologie structurale deux. Paris, Plon, 1973: 139-173. Jean-Claude Schmitt a préfacé, avec Daniel Fabre, la traduction française de Vladimir Propp: Les Racines historiques du conte merveilleux. Paris, Gallimard, 1983: VII-XXII.

8. Centre Louis-Gernet de recherches comparées sur les sociétés anciennes (CNRS-EHESS), créé en 1964 par Jean-Pierre Vernant.

9. Grand intellectuel, Antoine Lion (1940-2012) est entré dans l'ordre des Dominicains après des études à l'École polytechnique. Contre sa hiérarchie, il a combattu toutes les formes de discriminations en fondant, en particulier, Chrétiens \& Sida, qu'il comparait volontiers à Act Up.

10. Jean-Claude Schmitt (dir.), Eve et Pandora: la création de la première femme. Paris, Gallimard, 2002 par les spécialistes de la «grande » littérature. Ils m'ont amené à m'interroger sur les rapports entre oral et écrit et entre folklore et culture savante. Ils m'ont conduit vers les ethnologues de l'Europe, en premier lieu Daniel Fabre. Je l'ai rencontré à l'occasion du colloque international d'anthropologues et d'historiens sur le charivari, organisé avec le soutien de Jacques Le Goff. Daniel m'a notamment permis de repérer les ethnologues qui pouvaient être intéressés. Il a été mon interlocuteur principal au carrefour des deux disciplines, d'autant mieux qu'il était historien dans l'âme. Je n'imaginais pas que notre amitié dût s'interrompre aussi brutalement.

Dans la masse considérable des exempla médiévaux, certains apparaissaient comme les plus anciennes versions attestées de «contes-types ", toujours en circulation aujourd'hui. L'un d'eux, édité dès 1872, a attiré mon attention: il était dû au prédicateur et inquisiteur dominicain lyonnais Étienne de Bourbon, vers 1260, qui y décrivait un culte bizarre qu'il s'employa lui-même à éradiquer. Une note de l'éditeur du XIXe siècle laissait entendre que des traces de ce culte subsistaient sur place, dans un bois des Dombes. Je suis allé voir et... me suis converti à l'enquête ethnographique. J'ai étudié la légende étiologique du culte, Vladimir Propp ${ }^{7}$ à la main, j'ai lu Lévi Strauss et me suis plongé dans l'anthropologie des rituels. Entretemps, j'avais aussi suivi la formation à la recherche en anthropologie (FRA) de I'EHESS, qui m'avait permis de faire la connaissance de Marc Augé, Maurice Godelier, Claude Macherel, Françoise Zonabend, Martine Segalen et bien d'autres.

\section{G. - Quel rôle ont joué les antiquisants dans cette orienta- tion anthropologique?}

J.-C.S. - Là encore, le «hasard » a bien fait les choses! Pauline Schmitt Pantel, ma femme, avait été l'élève de Pierre Vidal-Naquet à Lyon. À la création de l'université de Paris VII, il lui a proposé d'y devenir assistante. Rapidement, nous avons fait la connaissance de Jean-Pierre Vernant qui, avec Le Goff, est devenu une de mes références intellectuelles majeures. Mais j'ai connu aussi tous les membres du Centre Louis-Gernet ${ }^{8}$ et on a beaucoup travaillé ensemble, notamment sur les images. Je ne me suis jamais senti historien de l'art, au sens - un peu caricatural, je l'avoue - où il m'intéresse moins d'identifier un artiste ou un atelier, de définir un style ou de proposer une nouvelle datation pour une œuvre que de réfléchir avec les «antiquistes » sur la place des images dans les rituels. Un lieu a beaucoup compté pour nos rencontres: le Centre Thomas-More, dans le couvent dominicain de La Tourette, construit par Le Corbusier à l'Arbresle, près de Lyon. Le père Ducret y a attiré Vernant et son équipe; après lui, Marc Augé en a présidé le conseil scientifique; je lui ai succédé quand il est devenu président de l'EHESS, tandis que la direction du Centre était échue à Antoine Lion, une personnalité inoubliable ${ }^{9}$. À l'Arbresle, je retrouvais les amis antiquistes François Lissarrague, Françoise Frontisi, Alain Schnapp, Jean-Louis Durand (lequel était aussi anthropologue au Burkina Faso). Notre bande d'amis, à laquelle se joignaient nos étudiants, s'y retrouvait durant plusieurs jours intensifs pour travailler sur les mythes (avec Jean-Pierre Vernant, Jean Bottéro, Marcel Détienne), les rites (avec Pierre Vidal-Naquet, Charles Malamoud) et, toujours et encore, les images. Le volume collectif Ève et Pandora que j'ai publié chez Gallimard 10 est né de l'une de ces rencontres. 
G. - En 2002, vous avez publié Le Corps des images: essais sur la culture visuelle au Moyen Âge ${ }^{11}$. Comment un médiéviste peut-il travailler avec des historiens de l'art? À quelles conditions la rencontre peut-elle se faire sur la question des images?

J.-C.S. - Je suis arrivé aux images par Vernant, et par des philosophes et sémiologues qui avaient une démarche très différente de celle de l'histoire de l'art traditionnelle: Hubert Damisch et Louis Marin m'ont appris à regarder les images, tout comme Jean-Claude Bonne, également philosophe de formation, qui a fait sa thèse sur la sculpture romane sous la direction de Damisch. J'ai commencé à travailler avec Jean-Claude Bonne en $1975^{12}$. Quand je suis devenu directeur d'études en 1983, j'ai décidé de consacrer mon séminaire aux images, avec l'idée de créer au GAHOM ${ }^{13}$ un groupe de recherche s'appuyant sur la constitution, par les chercheurs et les étudiants, à des fins pédagogiques autant que documentaires, d'une base de données iconographique. À l'époque, il était encore très difficile de rassembler une documentation massive dans ce domaine. Au CNRS, I'IRHT ${ }^{\mathbf{1 4}}$ avait commencé à photographier (sous forme de diapositives, qui aujourd'hui sont toutes numérisées et disponibles en ligne) et à indexer sommairement les fonds de manuscrits enluminés des bibliothèques de province. Notre but était différent: nous ne visions pas l'exhaustivité sur le territoire national, mais voulions acquérir des reproductions de manuscrits conservés à l'étranger, en Europe et aux États-Unis, autant qu'en France, utiles aux recherches des étudiants et des enseignants-chercheurs. Depuis lors, le travail d'indexation des miniatures se poursuit avec les étudiants, au gré de séances hebdomadaires qui sont aussi de grands moments de sociabilité et de libre discussion. Sous la conduite de Jérôme Baschet, un thesaurus de description iconographique a été mis au point et étendu à l'analyse des peintures murales et des sculptures par le Centre d'études supérieures de civilisation médiévale (CESCM) de Poitiers. Le GAHOM dispose à ce jour d'une base de près de quatre-vingt-dix mille reproductions précisément indexées. Cela représente une capacité documentaire inimaginable il y a trente ans. Mais l'essentiel pour moi a toujours été la vertu pédagogique de l'indexation collective avec les étudiants.

\section{G. - Sur quelles options théoriques se fondait la rupture avec les historiens classiques de l'art médiéval?}

J.-C.S. - L'approche anthropologique représente une rupture. Elle conditionne un élargissement considérable du champ. L'image n'est pas seulement matérielle et sensible, mais mentale: mémorielle, visionnaire, onirique. Voyez le vocabulaire: le mot imago désigne d'abord la catégorie première de l'anthropologie médiévale, l'homme créé «à l'image de Dieu ${ }^{15}$ ». La première fois que l'homme est mentionné dans la Genèse, il est nommé «image ». La légitimation de l'image chrétienne passe aussi par l'Incarnation: le Fils, qui est lui-même désigné comme «image du Père», s'est fait homme. Le lien judaïque entre l'homme et Dieu s'en est trouvé bouleversé et avec lui le statut des images et les conditions de leur légitimité. Prendre les choses de cette manière invite déjà à modifier ses stratégies de recherche à l'égard des images matérielles. Du reste, le seul fait de parler à leur propos d'«images", et non d' ' art ", a en soi des implications importantes: cela signifie que toutes
11. Jean-Claude Schmitt, Le Corps des images: essais sur la culture visuelle au Moyen Âge. Paris, Gallimard, 2002.

12. À cette date Jacques Le Goff ouvrait un deuxième séminaire à I'EHESS sur les images de la royauté, en collaboration avec Jean-Claude Bonne, Jean-Claude Schmitt et Michel Pastoureau.

13. Le Groupe d'anthropologie historique de l'Occident médiéval (GAHOM) fut fondé en 1978 par Jacques Le Goff au sein du Centre de recherches historiques (EHESS/CNRS).

Jean-Claude Schmitt l'a dirigé de 1992 à 2014. Site Internet: http://gahom. ehess.fr/

14. Institut de recherche et d'histoire des textes, laboratoire propre du CNRS.

15. Jean-Claude Schmitt, "La culture de l'imago ", Annales HSS 51 (1), 1996 : 3-36. 
16. La collection

"Le temps des images", que Jean-Claude Schmitt et François Lissarrague ont dirigée pendant une vingtaine d'années aux éditions Gallimard, a publié deux ouvrages de Hans Belting: Pour une anthropologie des images, trad. de l'allemand par Jean Torrent, 2004, et La Vraie Image: croire aux images?, trad. de l'allemand par Jean Torrent, 2007.

17. Trad. de l'allemand par Frank Muller, Paris, éditions du Cerf, 1998. La page de titre reproduit fidèlement le titre allemand: Bild und Kult: eine Geschichte des Bildes vor dem Zeitalter der Kunst. Beck, München, 1990, mais le titre français en couverture est erroné.
18. Alphonse Dupront, L'Image de religion dans l'Occident chrétien: d'une iconologie historique, préface de Mona Ozouf. Paris, Gallimard, 2015.

19. Voir Pierre Francastel, "Art et hérésie", in Jacques Le Goff (dir.), Hérésies et sociétés dans l'Europe préindustrielle $\left(x I^{e}-x V I I I^{e}\right.$ siècles), actes du Colloque de Royaumont (1962), Mouton \& Cie, 1968: 31-50.

20. Voir Jean-Claude Schmitt, "Unorthodox" Images? ", in Sophie Page (dir.), The Unorthodox Imagination in Late Medieval Britain, actes du colloque «Neale Lecture ", University College de Londres (2006). Manchester/New York, Manchester University Press/Palgrave Macmillan, 2010: 9-38. ci-contre

fig. 1

Psautier dit de saint Louis, 1270-1274. Le manuscrit est un psautier à l'usage de Paris et plus spécialement de la Sainte-Chapelle, dont toutes les fêtes sont mentionnées dans le calendrier. Paris, Bibliothèque nationale de France, Ms Latin 10525, folio $13 \mathrm{v}$. les images (même les plus modestes, les moins "belles", sans auteur connu), que les séries dans lesquelles le chercheur assigne une place à ces images, que les usages et fonctions de ces images et séries d'images, que tout cela est intéressant et porteur de sens pour l'historien.

G. - Vous avez aussi défendu l'idée, par rapport à Hans Belting, qu'il fallait considérer les dimensions cultuelle et esthétique comme des polarités, plutôt que de les opposer comme des périodes historiques de l'iconographie religieuse?

J.-C.S. - Hans Belting, dont j'ai œuvré à la traduction de plusieurs livres en France ${ }^{\mathbf{1 6}}$, a volontairement rompu avec la tradition de l'histoire de l'art, y compris dans sa carrière, en délaissant la prestigieuse chaire d'histoire de l'art de Munich au profit d'un enseignement à l'École d'art et de design de Karlsruhe. Le titre d'un de ses livres les plus connus, Image et culte: une histoire de l'image avant l'époque de l'art 17, pourrait faire penser que la conception anthropologique de l'image (Bild) n'aurait plus lieu d'être à partir de la Renaissance et de la création d'un "art » (Kunst) affranchi des contraintes religieuses médiévales. Au début des Temps modernes - une période que Belting n'évoque qu'en conclusion - se produisit selon lui une "crise de l'image" (au sens de l'imago médiévale). Mais les usages cultuels de certaines images n'ont pas disparu pour autant, comme l'a montré Alphonse Dupront ${ }^{18}$.

\section{G. - II y a un autre historien de l'art auquel vous vous référez volontiers, c'est Pierre Francastel.}

J.-C.S. - Francastel a été le maître à penser de Hubert Damisch. Cette filiation entre Francastel-Damisch-Bonne et aussi Le Goff, qui l'admirait beaucoup, a été importante. Les travaux de Francastel sur la sociologie de l'art et sur la perspective demeurent fondamentaux. Lors du colloque “Hérésie et société» de 1962, organisé par Jacques Le Goff, Francastel a posé une question à mon sens cruciale sur les rapports entre image et hérésie ${ }^{19}$ : au Moyen Âge, le soupçon de l'hérésie se porte sur les textes et la parole publique, et non sur les images. Les usages impropres et symétriquement opposés des images (l'iconoclasme d'un côté, l'idolâtrie de l'autre) sont étroitement surveillés et le cas échéant sanctionnés, tandis que, jusqu'au $X V$ siècle, ce n'est pas le cas de l'iconographie, dont les innovations sont parfois, pourtant, d'une grande hardiesse 20.

\section{G. - Pouvez-vous préciser cette distinction entre la sanction} sur les usages des images et la sanction sur l'image elle-même?

J.-C.S. - Cela commence dès les Pères de l'Église, repris à l'époque carolingienne en réponse à la crise iconoclaste byzantine. Lors du rétablissement en Orient du culte des icônes, le pape Adrien se fait auprès de Charlemagne l'avocat de l'iconodoulie des Grecs. Les Libri Carolini expriment la position plus modérée de l'entourage du roi franc, qui reste fidèle à l'enseignement de Grégoire le Grand: il faut respecter les images, ne pas les détruire, mais pas non plus les «adorer». Cette défense d'une voie moyenne vise aussi, en sens inverse, les partisans occidentaux de l'iconoclasme, 


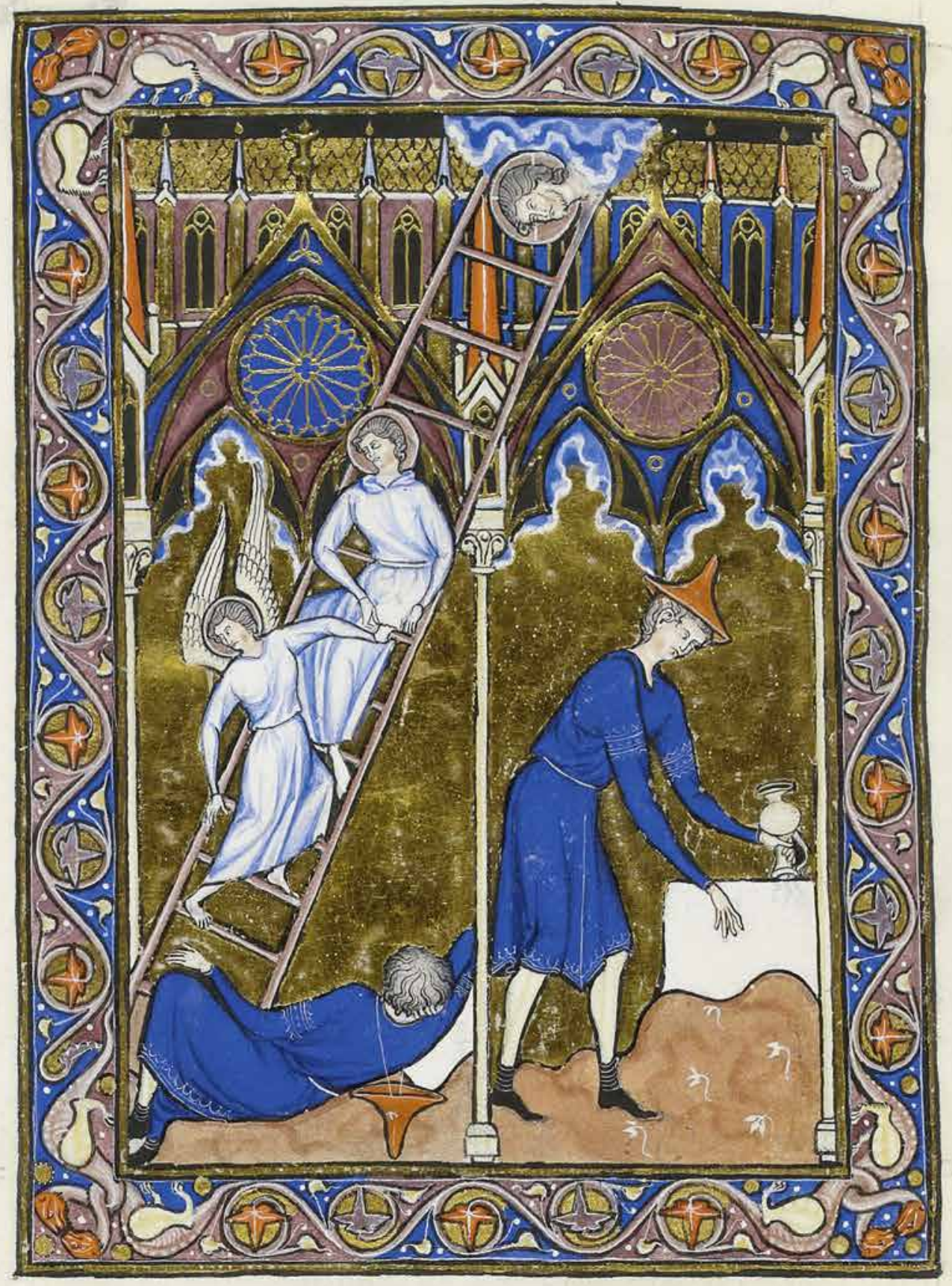


21. Voir Jean-Claude Schmitt, La Conversion d'Hermann le Juif: autobiographie, histoire et fiction. Paris, Seuil, 2007 [2003].

22. Voir, en particulier, les articles rassemblés dans la section III, "Le sujet et ses rêves", in Le corps, les rites..., op. cit. : 240-315; pour l'ethnologie, voir Terrain, "Rêver ", 26, 1996.

23. Gisèle Besson et Jean-Claude Schmitt (trad. et éd.), Rêver de soi: les songes autobiographiques au Moyen Âge. Toulouse, Anacharsis, 2017 tel l'évêque Claude de Turin. Cependant, en Occident aussi, l'idée s'impose que l'image est le médium matériel du transitus vers le divin et cette évolution coïncide avec la généralisation des images cultuelles en trois dimensions: crucifix, statues de la Vierge et des saints. La "Majesté de sainte Foy " de Conques, qui est à la fois une statue et un reliquaire, est l'un des exemples les plus connus de ces «nouvelles images", vers l'an mil. Or la troisième dimension caractérisait traditionnellement les idoles païennes: elle fait scandale, avant d'être définitivement acceptée. Au XII ${ }^{\mathrm{e}}$ siècle, la question est encore posée dans les débats - parfois fictifs - entre juifs et chrétiens au sujet des images. Les premiers sont censés accuser les seconds d'«adorer» les images, ce qui permet aux clercs d'argumenter en faveur du culte, légitime à leurs yeux, du crucifix et des images des saints ${ }^{21}$.

G. - On vous doit, aussi, la découverte des images de rêve et de vision, et toute une réflexion qui rejoignait les préoccupations des ethnologues, sur la distinction entre image mentale et image matérielle 22 .

J.-C.S. - Je viens de publier avec Gisèle Besson une anthologie de rêves autobiographiques médiévaux, Rêver de soi ${ }^{\mathbf{2 3}}$. II existe très tôt, dès le $\|^{\mathrm{e}}$ siècle, des récits «autobiographiques » de rêve - je pense au témoignage laissé par la martyre sainte Perpétue. Mais c'est surtout à partir du Xle siècle, dans les milieux monastiques, que l'on commence à transcrire et commenter ses propres rêves et que l'on ne se contente plus de faire l'exégèse des rêves bibliques de Joseph ou de Jacob. En revanche, il semble que jamais personne au Moyen Âge n'ait peint ses propres rêves, alors que les miniatures et les sculptures représentent à foison les rêves bibliques ou d'autres rêves littéraires. Le premier peintre qui a figuré son rêve personnel est Dürer: s'éveillant terrorisé en plein cauchemar, il décide sur-le-champ de décrire celui-ci et d'en faire une aquarelle sur la même feuille, qu'il date et signe. Cette image innove aussi par le fait que le rêveur ne s'est pas représenté en train de rêver, à l'inverse de toutes les images médiévales de rêve, où l'on voit par exemple Jacob endormi au pied de l'échelle céleste dont il rêve (fig. 1). L'image médiévale juxtapose et associe de manière subtile le sujet et l'objet du rêve. Dürer rompt pour la première fois avec cette tradition en ne figurant que l'objet du rêve.

\section{G. - Ce numéro de Gradhiva traite de pratiques cultuelles contemporaines en privilégiant, plutôt que les valeurs, les modes d'engagement du corps, les régimes sensoriels qu'elles con- voquent. Qu'en pense le médiéviste qui nous a fait découvrir la richesse de ces questionnements pour les sociétés médiévales?}

J.-C.S. - Tout m'a passionné dans ce numéro, qu'il s'agisse des questions de calligraphie et d'ornementation, ou encore des technologies télévisuelles liées à la prédication, sachant que le rôle du prédicateur dans l'espace public est une question centrale depuis le XIII ${ }^{e}$ siècle; il en va de même des écritures pictographiques à trois dimensions qui suggèrent des comparaisons possibles avec les diagrammes didactiques sur lesquels je travaille en ce moment pour la période médiévale. La «lecture» de ces diagrammes, qui sont très présents dans les manuscrits, engage le corps en 
imposant un parcours visuel et mnémotechnique entre tous les éléments qui composent la figure. Certains de ces diagrammes soutiennent la réflexion astronomique, d'autres, de contenu moral ou théologique, peuvent être associés à la prédication. Certains sont des dessins au trait, très simples, utilisés par les prédicateurs, d'autres, comme ceux du Vergier de Soulas, se parent de couleurs et d'un fond d'or pour l'édification morale d'une destinataire aristocratique. Chaque figure - croix, arbre, roue, château, etc. possède son propre sens de déchiffrement, de bas en haut et de gauche à droite, ou de façon circulaire, souvent balisé par la succession des lettres de l'alphabet. D'une lettre à l'autre, des personnages bibliques ou des saints apparaissent, avec des citations des Écritures à mémoriser. Dans la «Tour de la sagesse", toutes les pierres de l'édifice sont identifiées par des lettres différentes qui renvoient aux dix commandements, aux sept pétitions du Notre Père, aux trois vertus théologales et aux quatre vertus cardinales, etc. Les saints - Grégoire, François, Benoît - couronnent la tour crénelée. Pour sa part, la «Roue de la foi » (fig. 2) affiche en son centre son mode d'emploi, avec des lettres qu'on retrouve au début des inscriptions courant sur les cercles concentriques et de nouveau sur les phylactères portés par les personnages de la bordure: on commence par lire à partir d'une petite croix la première pétition du Notre Père, puis on se reporte au premier saint de la bordure; après quoi, on revient à la deuxième section du premier cercle, puis au deuxième saint et à son phylactère, et ainsi de suite. Ce mouvement contraint des yeux, ce va-et-vient entre centre et périphérie et cette progression circulaire d'un segment à l'autre de la roue, depuis le cercle extérieur jusqu'au centre, suppose une discipline corporelle qui soutient le travail de la mémoire et aide à l'inculcation de la croyance et des règles morales. II faut de plus imaginer une lecture vocalisée, à voix haute ou marmonnée, exactement comme dans la récitation contemporaine du Rosaire. Nous avons là un chapelet en image.

G. - Face à ces images, le lecteur pourrait-il se transformer en visionnaire? Pourrait-il, en suivant ce cheminement, arriver à la vision du saint ou de Dieu?

J.-C.S. - Peut-être pas avec ces diagrammes et dans ce milieu, mais sans doute dans les Rothschild Canticles, un manuscrit du XIV siècle édité par Jeffrey Hamburger ${ }^{\mathbf{2 4}}$. Ce manuscrit de très petite taille devait être en usage dans une communauté de béguines mystiques. Un des cycles est consacré à La Trinité, qui est l'objet d'une métamorphose visuelle dont toutes les étapes s'enchaînent dans les enluminures du manuscrit, jusqu'à l'illumination finale où l'on ne voit plus qu'une explosion de lumière. Tout cela est censé se produire dans le cœur du Christ de la Passion; il indique lui-même de la main à la mystique, ou plutôt à son âme représentée sous les traits d'une femme armée d'une lance, la plaie du côté par lequel elle doit pénétrer de force pour aller se fondre dans le cœur divin. L'image la représente ensuite en extase devant La Trinité qui lui tombe littéralement dessus. On dirait la sainte Thérèse du Bernin! Puis La Trinité anthropomorphe commence sa métamorphose, et l'on voit les ailes de la colombe du Saint-Esprit se transformer en sortes de voiles. Et les personnes divines s'abîment dans l'éblouissement final, tel que la mystique pouvait l'éprouver (fig. 3,4 et 5) ...
24. Jeffrey Hamburger, The Rothschild Canticles: Art and Mysticism in

Flanders and the Rhineland circa 1300. New Haven/

Londres, Yale University

Press, 1990. Voir aussi

Jean-Claude Schmitt,

"Les exempla et les

images dans les Rothschild

Canticles et le Ci nous dit

(début du XIV ${ }^{\mathrm{e}}$ siècle)"

in René Wetzel, Patrice

Flückiger (dir.),

Die mittelalterliche Predigt

zwischen Mündlichkeit,

Bildlichkeit und

Schriftichkeit /

La prédication au Moyen

Age entre oralité, visualité

et écriture. Zürich,

Chronos, 2010: 157-176. 


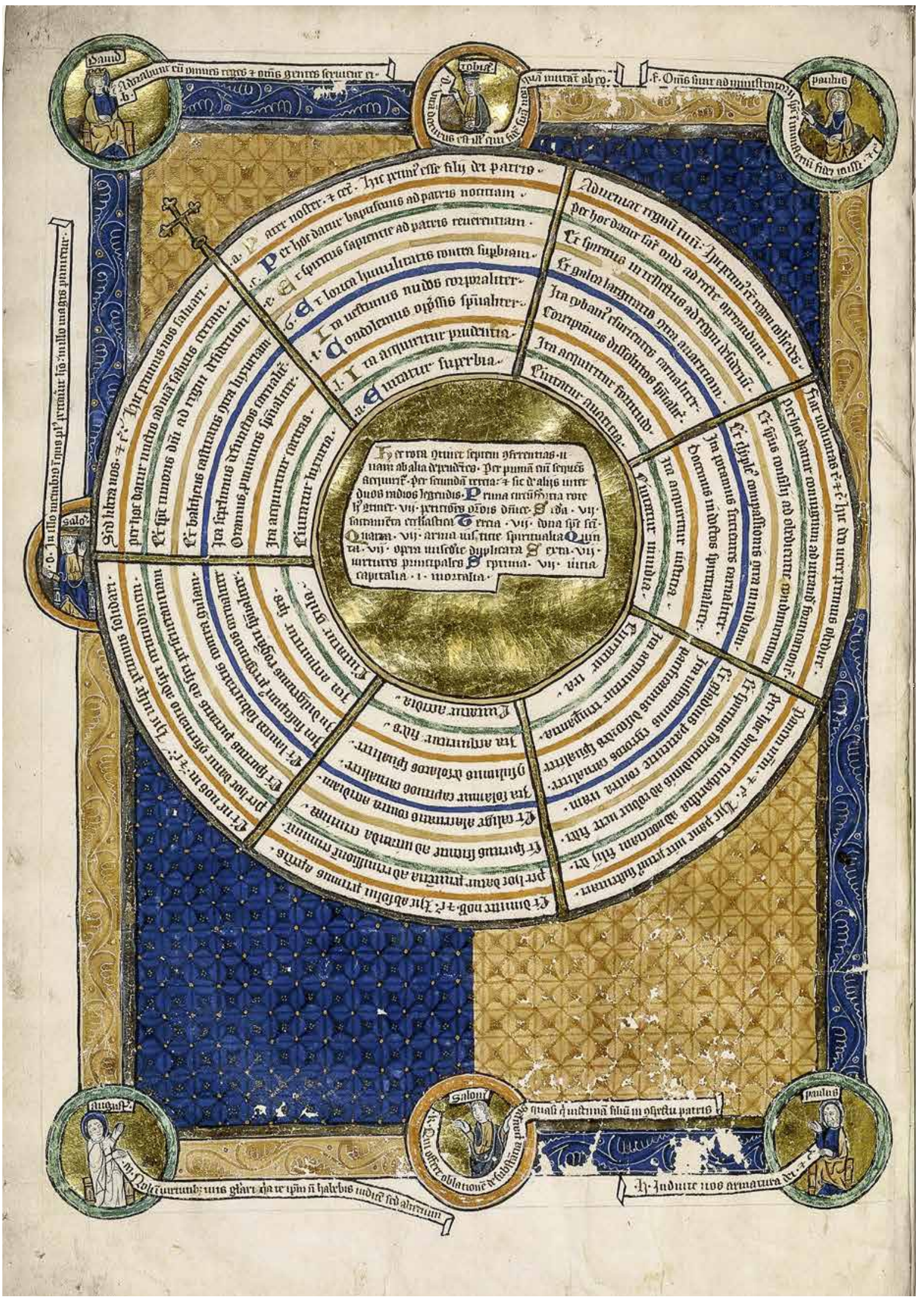


G. - Cet effacement progressif des figures anthropomorphes semble confirmer ce que vous montrez dans Les Rythmes au Moyen Âge 25 : les artistes médiévaux inventent, d'une certaine façon, l'abstraction ou, en tout cas, se posent des questions analogues à celles des avant-gardes $d u x^{e}$ siècle, comme le suggèrent vos références à Klee, à Kandinsky et aux grands historiens de l'art allemand...

J.-C.S. - En effet, l'art abstrait contemporain est profondément rythmique. Les images médiévales, dans leur usage des formes et des couleurs, ne le sont pas moins. Je suis depuis longtemps sensible aux affinités plastiques entre ces deux mondes iconiques, si différents à bien des égards. Le rythme des images provient aussi de leurs conditions d'usage: progresser dans la lumière des vitraux d'une cathédrale (sans qu'il soit besoin de les déchiffrer), tourner les pages d'un manuscrit enluminé durant la liturgie crée du rythme en faisant miroiter l'or et les couleurs et rend indissociables les expériences esthétique et religieuse. C'est l'une des nombreuses raisons pour lesquelles je me suis passionné pour l'étude des rythmes. Je leur ai consacré de nombreuses années de travail, d'autant plus qu'il n'existait pas d'étude historique sur cette question; celle-ci, en revanche, avait fondé le programme sociologique et anthropologique d'un Marcel Mauss, qui voyait dans les rythmes sociaux le principe des catégories du temps en vigueur dans chaque civilisation. Plus récemment, Gilles Deleuze et Félix Guattari ont impulsé une réflexion renouvelée sur les rythmes. Mais les historiens n'ont pas suivi. Il est vrai que la question est complexe, parce qu'elle touche à tous les aspects de la vie individuelle et collective. Trouver le plan de mon livre n'a pas été facile: j'ai finalement pris le parti de donner à l'ouvrage le rythme même des six jours de la création du monde selon la Genèse! Ne vivonsnous pas aujourd'hui encore au rythme biblique de la semaine? Saint Augustin $y$ avait vu aussi le modèle d'autres rythmes fondamentaux: les «âges du monde" scandant l'histoire universelle, aussi bien que les «âges de la vie" à travers lesquels chaque individu est invité à passer. Dans ce livre, les images et les séries d'images sont très présentes, car les rythmes visuels sont fondamentaux, tout autant que les rythmes sonores (ceux de la danse, du chant liturgique, des cloches, etc.). La régularité, la périodicité sont des composantes de la notion de rythme, mais les variations, les silences, en un mot l'arythmie, ne le sont pas moins. Le rythme n'est jamais pure répétition mécanique. Le jazz, voilà le rythme. Et du jazz je rapprocherais volontiers les images médiévales, d'autant que pour moi elles sont aussi musique: elles chantent, pour ainsi dire, et beaucoup d'entre elles sont destinées au monde éminemment musical de la liturgie; n'oublions jamais que la musica, c'est l'ordre divin du monde et donc aussi de sa mise en image ${ }^{26}$.

\section{G. - S'agissant de l'ornementation des livres saints, une comparaison est-elle possible entre l'ornementation du Coran et celle de la Bible dans le christianisme latin?}

J.-C.S. La calligraphie est importante dans ces deux cultures, particulièrement dans I'Islam, par principe hostile à la figuration de Dieu et du prophète. La minorité chiite accepte toutefois de représenter le prophète Mohammed, le visage le plus souvent voilé, et plus ouvertement, son gendre
25. Jean-Claude Schmitt Les Rythmes au Moyen Âge. Paris, Gallimard, 2016.

26. Voir Jean-Claude Schmitt, "La musique des images ", in Martine Clouzot, Christine Laloue et Isabelle Marchesin (dir.), Moyen Âge: entre ordre et désordre, catalogue d'exposition (Paris, musée de la Musique, 2004) Paris, Musée de la Musique/RMN, 2004 : 44-51.
ci-contre
fig. 2
Le Verger de Consolation, vers 1290. La roue des correspondances théologiques. Paris, Bibliothèque nationale de France, Ms Français 9220, folio $11 \mathrm{v}$.









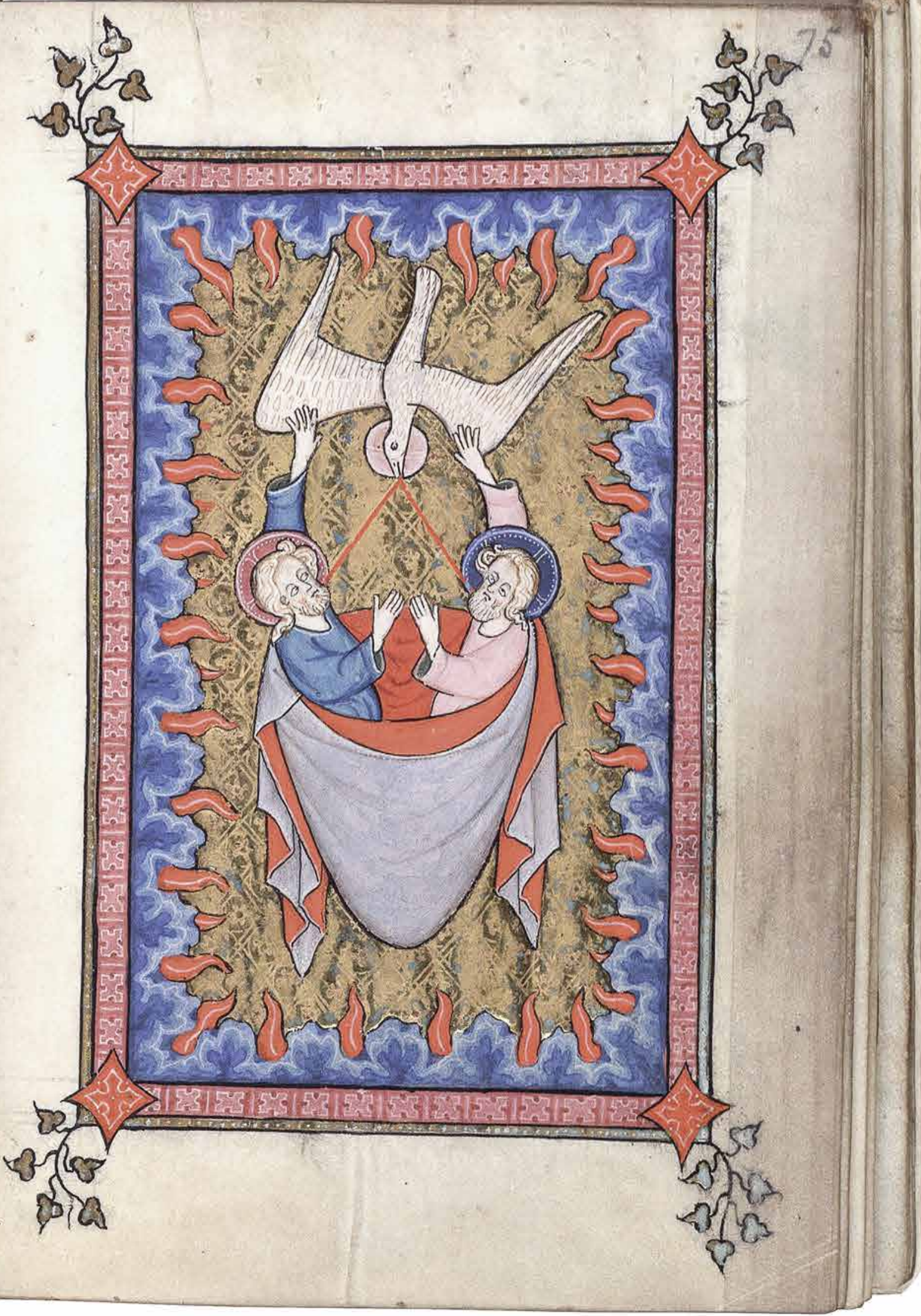


27. Voir Les Rythmes au Moyen Âge, op. cit. : 199-201.

28. Mary Carruthers, Le Livre de la mémoire: une étude de la mémoire dans la culture médiévale, trad. de l'anglais par

Diane Meur. Paris, Macula, 2002. double page

précédente

fig. 3

Canticles La Trinité,

XIII-XIV ${ }^{e}$ s. New Haven, CT, États-Unis, Yale University, Beinecke Library, MS 404, folio $74 \mathrm{v}$ et $75 \mathrm{r}$.

\section{ci-contre}

fig. 4

Canticles La Trinité,

XIII-XIve s. New Haven, CT, États-Unis, Yale University, Beinecke Library, MS 404, folio $84 r$.
Ali, ainsi que le fils de celui-ci, Hussein. En Iran aujourd'hui, on voit beaucoup de «portraits» du Prophète en jeune homme, avant qu'il n'ait reçu la révélation du Coran. Le christianisme latin a lui aussi connu des hésitations, dont la scène du Buisson ardent est un bon révélateur: suivant la Genèse, Moïse aurait seulement perçu la voix de Yahvé, sans le voir. Pour évoquer la voix divine, les imagiers chrétiens n'hésitent pas à représenter la face de Dieu dans le Buisson ardent; mais ils le dotent d'un nimbe cruciforme, ce qui rappelle que c'est l'Incarnation du Christ qui rend légitime pour les chrétiens la figuration divine. Dans d'autres cas, le visage de Dieu est caché, recouvert d'un quadrilobe ou même retourné, comme si Dieu s'était montré de dos! Mais je reviens à l'écriture, à ses effets esthétiques, et aussi à la performance physique qu'elle représente pour le scribe au fil des heures et des journées: dans les manuscrits médiévaux les plus précieux, la calligraphie doit être parfaite et suppose donc de la part du scribe une maîtrise absolue de son art, un contrôle non seulement du geste, mais de la respiration qui le soutient, comme on a pu le démontrer par des mesures très fines des variations de l'épaisseur du trait et de l'espacement des lettres tracées par une même main ou par des mains différentes qu'on parvient ainsi à identifier. On a même pu repérer les moments où la fatigue gagnait le scribe et le contraignait à suspendre son travail ${ }^{27}$. C'est tout le corps et l'esprit qui participent au geste graphique, soumis de surcroît à un fort contrôle social, à l'intérieur du scriptorium monastique, et plus tard en ville chez les stationarii qui recopiaient les peciae universitaires. À l'écriture s'ajoute l'ornementation, ce qui vaut aussi pour les manuscrits du Coran. On s'interroge sur le partage des tâches entre scribe et peintre, sur l'ordre de la chaîne opératoire, puis sur les fonctions de l'ornementation et de l'iconographie dans la transmission du texte: pour Mary Carruthers, les motifs floraux et les marges peuvent aider à la mémorisation du message ${ }^{\mathbf{2 8}}$, sans qu'on puisse les assimiler pour autant aux «arts de la mémoire» antiques.

G. Comparer les rituels d'image télévisuelle des pentecôtistes d'aujourd'hui et ceux propres à l'imago médiévale vous paraît-il légitime? Peut-on, par exemple, les rapprocher de cette chaîne des regards qui fait "entrer dans l'image ", selon l'expression que vous employez dans Le Corps des images, à propos d'une miniature figurant saint François d'Assise, à genoux, contemplant le séraphin et recevant les stigmates, tandis que Hailwigis, une petite nonne dominicaine, contemple le saint?

J.-C.S. - II est très facile de trouver, tout en maintenant la distance nécessaire, des situations comparables d'utilisation conjointe de la parole et de l'image, notamment par les prédicateurs. Pensez à Bernardin de Sienne exhibant en chaire sa fameuse tablette frappée du monogramme de Jésus devant la foule fervente amassée sur la place publique. Ce type de «performance" fait appel simultanément aux sens de la vue et de l'ouïe. Bien sûr, il n'y a pas de télévision au Moyen Âge, mais la recherche du contact par la vue et même le toucher de l'image réputée miraculeuse est bien attestée. La miniature de saint François que vous évoquez appartient à un manuscrit de prières monastiques. La petite religieuse Hailwigis, comme d'autres sœurs également nommées, a tenu ce livre entre ses mains: elle s'est donc vue en train de voir, se voyant dans l'image en train de voir Dieu à travers 


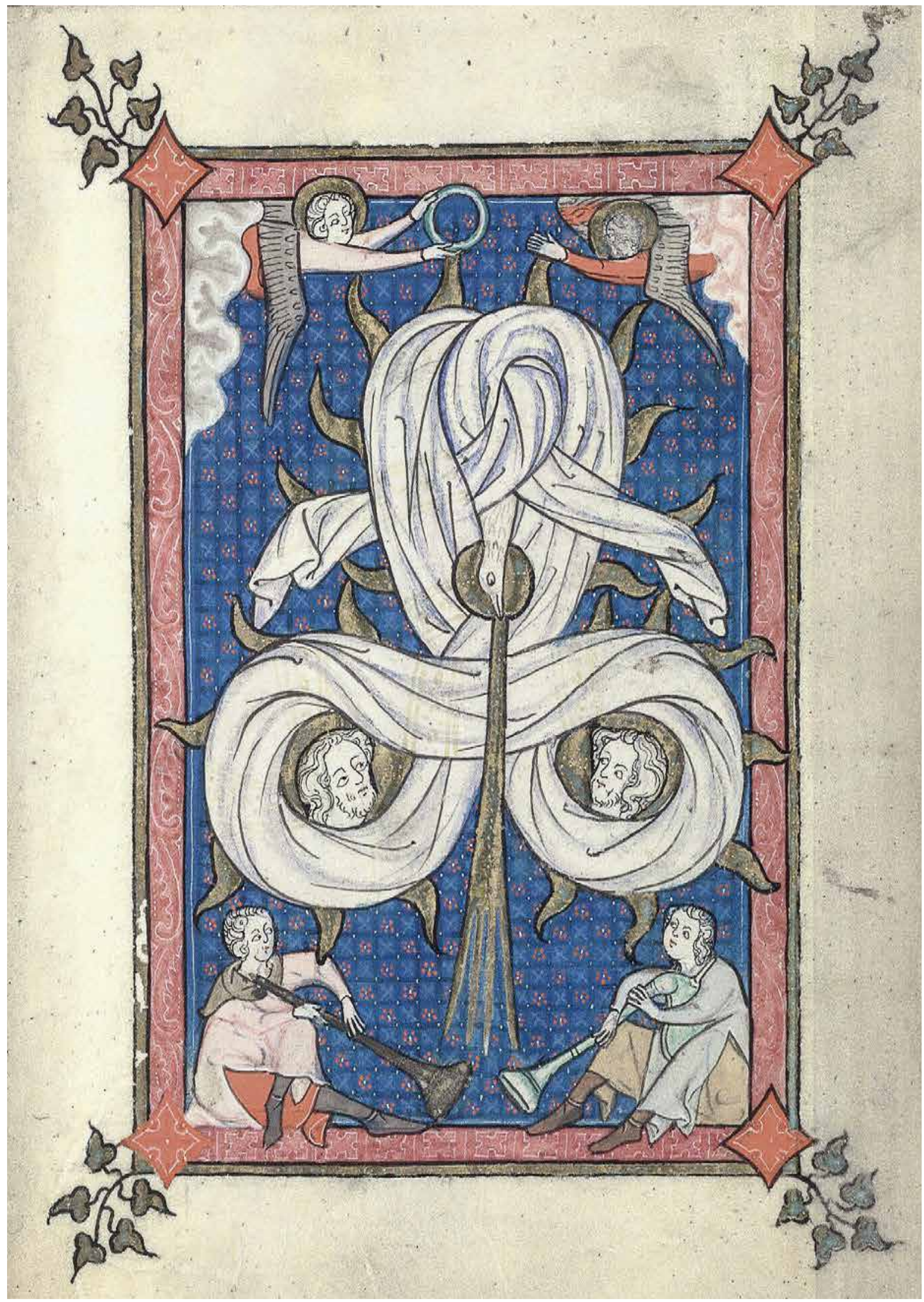




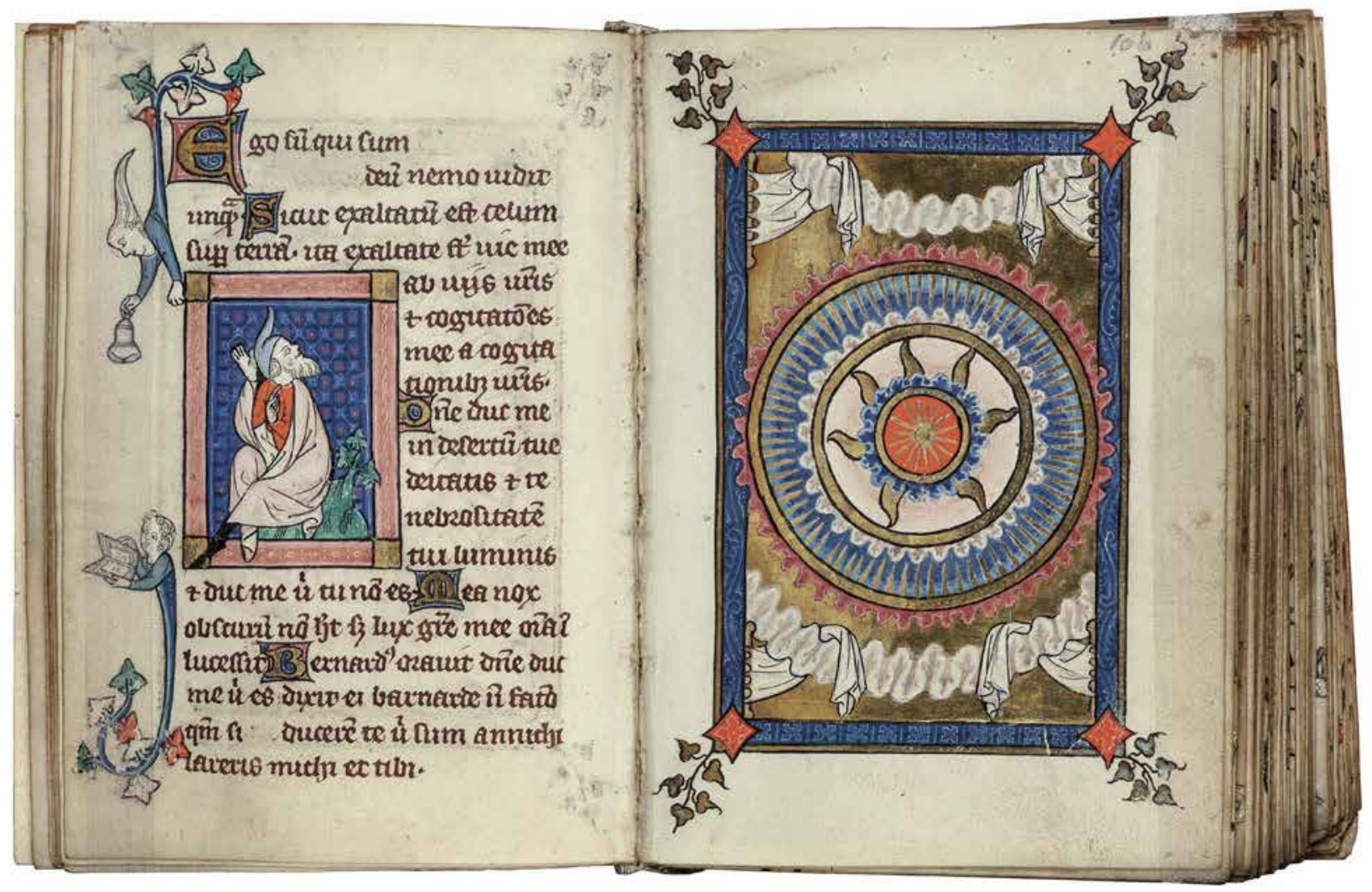

ci-dessus et

p. 164

fig. 5

Canticles La Trinité,

XIII-XIV' S. New Haven, CT, États-Unis, Yale University, Beinecke Library, MS 404, folio $105 \mathrm{v}$ et $106 \mathrm{r}$. l'image et par la grâce de sa prière. Une telle image fonctionne de manière réflexive. Aujourd'hui, dans certaines églises de pèlerinage, on voit des dévots faisant avec leur téléphone portable des selfies sur fond de statue miraculeuse de la Vierge. On pourrait multiplier les points de comparaison: bien des images médiévales me paraissent proches de nos images publicitaires, non seulement par la fonction de «propagande» (commerciale pour celles-ci, religieuse pour celles-là), mais aussi par leurs caractéristiques formelles, le rapport entre la figure et le fond, la simplicité de la structure, un certain schématisme, le contraste affirmé des couleurs, le traitement du temps et du rythme, l'inclusion de textes brefs dans l'image, toutes choses qui contribuent à l'efficacité du message. Et celui-ci, dans tous les cas, fait s'ouvrir l'image sur l'imaginaire et sur le désir, quel qu'en soit l'objet.

G. Ce n'est pas sans inquiétude que certains anthropologues se tournent vers les travaux des médiévistes, pour mieux percevoir la spécificité des enjeux contemporains. Que pensez-vous de ces sauts dans le temps?

J.-C.S. - II y a en effet aujourd'hui chez les anthropologues un grand besoin de profondeur historique, d'autant plus que l'anthropologie comme discipline a désormais sa propre histoire, qui ne cesse de s'allonger. Histoire et anthropologie usent l'une et l'autre du dépaysement que provoque l'écart dans le temps et/ou l'espace, sans que l'on puisse continuer de dire que 
l'axe temporel est l'apanage de l'historien, tandis que l'axe spatial serait celui de l'anthropologue féru de comparatisme. Les deux sont confrontés aussi aux risques apparentés de l'ethnocentrisme ou de l'anachronisme, mais ils ont appris à les maitriser à des fins de compréhension et de démonstration. Plus nous contrôlons la distance qui nous sépare de notre objet, mieux nous pouvons en jouer pour comprendre et faire comprendre la spécificité de ce dernier ${ }^{29}$. La question des mots dont nous usons dans nos analyses est essentielle et nous en parlions au début de cet entretien: j'aime bien provoquer en disant qu'on ne peut parler de «religion » au Moyen Âge! J'entends par là que le système de représentation et de croyances associant l'ici-bas - le social et le politique - et l'au-delà était complètement intégré dans ce qu'on peut appeler la «chrétienté » (la totalité du système social dont le christianisme est l'idéologie), aux antipodes de la conception moderne de la religion comme option individuelle et comme segment particulier de la culture. Étudier la chrétienté exige de l'historien une mise à l'écart de ses manières habituelles de penser et l'invite à faire de l'anthropologie. En 2013, je suis allé observer le Círio de Notre-Dame de Nazaré, l'immense pèlerinage marial qui se déroule chaque année dans la ville de Belém au Brésil, le deuxième dimanche d'octobre ${ }^{30}$. Deux millions de personnes viennent de partout pour suivre en procession une toute petite statue de la Vierge. Je me suis intéressé aux effets du dédoublement de la statuette, intervenu il y a une vingtaine d'années: désormais, l'image réputée "originale» demeure dans son sanctuaire, tandis que sa copie, portée en procession à travers la ville, entraîne derrière elle une foule immense et exaltée. La copie a sur l'originale le grand avantage de se mouvoir! Toutefois, la «vraie » Vierge - que sa doublure n'a rencontrée à aucun moment - reçoit au final les ex-voto que les fidèles déposent en vrac dans son église, notamment des modèles réduits de chaises d'étudiant avec tablette pour ceux qui espèrent réussir le concours d'entrée à l'université, ou des maisonnettes en bois pour ceux qui comptent sur la Vierge pour mieux se loger. Aurais-je chaussé à Belém les lunettes de l'ethnologue si je n'avais pas été médiéviste? Je ne le crois pas, et je voudrais une fois encore plaider pour que les historiens et les anthropologues multiplient les occasions d'un regard croisé sur leurs objets respectifs.
29. Jean-Claude Schmitt a explicité quelques enjeux contemporains du dialogue entre historiens et anthropologues dans: "L'anthropologie historique de l'Occident médiéval: un parcours ", L'Atelier du Centre de recherches historiques. Revue électronique du $\mathrm{CRH} 6$ [en ligne], disponible sur: https://acrh.revues. org/1911

30. Voir Jean-Claude Schmitt, «The Two Bodies of the Virgin: On the

Festival of Círio de Nazaré (Belém, 13th October, 2013) " in Babette Hellemans (dir.), Degree Zero of Sound and Image: Creation Before the Act (ca. 1000-1800). Amsterdam, Amsterdam University Press (à paraître). 